\title{
In silico design of a drug from the compounds that are suggestive in the treatment of arsenicosis using molecular docking
}

\author{
Tarek G M Mohammod \\ Mir Misbahuddin \\ Department of Pharmacology \\ Arsenic Research Division \\ Bangabandhu Sheikh Mujib Medical University
}

\begin{abstract}
:
In our quest to find a cure for arsenicosis, an in silico attempt was made to identify a common target for the compounds that relieves the symptoms using Autodock and Discovery studio. Retinol, alphatocopherol, ascorbic acid, alpha-lipoic acid and folic acid were used as ligands and an enzyme telomerase reverse transcriptase which was selected as the target protein to search for the structure which had the highest binding affinity with this cancer causing enzyme of the human body. This compound was compared with other potential inhibitors currently under research by docking with 47 important enzymes of the human body found in the protein data bank. Assessment by FAF-Drugs web server showed this compound to be non-toxic. To prove the effectiveness of this drug, both in vivo and in vitro studies are required along with this in silico study.
\end{abstract}

Keywords: Arsenicosis, Autodock, Telomerase Reverse Transcriptase Introduction:

Cancer of skin along with cancer of some internal organs- liver, kidney, urinary bladder is not uncommon [7] in arsenicosis. Patients once affected may not recover even after remediation of the arsenic contaminated water. Several vitamins and minerals such as vitamin A [6], ascorbic acid [13], alpha-tocopherol [5], zinc [8], folic acid [11], alpha-lipoic acid [18], and selenium [16] used either alone or in combination were found to be effective in arsenicosis. Some of our foodstuffs such as

corn [4], spinach [3], garlic oil [10] and spirulina [9] were proven to relieve the symptoms of arsenicosis. It is still unknown where these compounds act inside the body. The active ingredient inside the foodstuffs responsible for the relief of symptoms is still not identified. The compounds retinol and alpha-tocopherol have specific proteins to which they bind in the serum. But there are no transport proteins for ascorbic acid, folic acid and alpha-lipoic acid. These compounds along with zinc and selenium may have an antioxidant effect in mitigating the oxidative stress produced by excessive arsenic in the body. This study has been undertaken to identify the sites where these compounds function and to recognize a common location where one drug designed from these compounds works.

Although modeling techniques for prediction of binding affinity are reasonably successful, there are many other properties, such as bioavailability, metabolic half-life, lack of side effects, etc., that first must be optimized before a ligand can become a safe and efficacious drug. These other characteristics are often difficult to optimize using rational drug design techniques. In addition, these drugs should also be designed so as not to affect any other important "off-target" molecules or antitargets that may be similar in appearance to the target molecule, since drug interactions with off-target molecules may lead to undesirable side effects. Sequence homology is often used to identify such risks. Structure-based drug design (or direct drug design) relies on knowledge of the 
three dimensional structure of the biological target obtained through methods such as $\mathrm{x}$-ray crystallography or NMR spectroscopy.

As experimental methods such as X-ray crystallography and NMR develop, the amount of information concerning 3D structures of biomolecular targets has increased dramatically. In parallel, information about the structural dynamics and electronic properties about ligands has also increased. This has encouraged the rapid development of the structure-based drug design. Current methods for structure-based drug design can be divided roughly into two categories. The first category is about "finding" ligands for a given receptor, which is usually referred as database searching. In this case, a large number of potential ligand molecules are screened to find those fitting the binding pocket of the receptor. This method is usually referred as ligand-based drug design. The key advantage of database searching is that it saves synthetic effort to obtain new lead compounds. Another category of structure-based drug design methods is about "building" ligands, which is usually referred as receptor-based drug design. In this case, ligand molecules are built up within the constraints of the binding pocket by assembling small pieces in a stepwise manner. These pieces can be either individual atoms or molecular fragments. The key advantage of such a method is that novel structures, not contained in any database, can be suggested [19; 14].

In contrast to traditional methods of drug discovery, which rely on trial-and-error testing of chemical substances on cultured cells or animals, and matching the apparent effects to treatments, rational drug design begins with a hypothesis that modulation of a specific biological target may have therapeutic value. Once a suitable target has been identified, the target is

normally cloned and expressed. The expressed target is then used to establish a screening assay. In addition, the three-dimensional structure of the target may be determined.

\section{Materials and Methods:}

Softwares:

a. Discovery Studio 3.5

b. AutodockVina

c. Marvin Sketch 6.1, 2013, ChemAxon (http://www.chemaxon.com)

d. Online software tools:

i. BLAST [2],

ii. ClustalW2,

iii. FAF-Drugs web-server [1] Molecular docking

Alpha-tocopherol was modified using discovery studio 3.5 to 289 structures and in silico docking study was done with the target protein using Autodock Vina to identify the structure with highest binding affinity. Naming of the compounds was done by Marvin Sketch 6.1, 2013, ChemAxon

\section{Assessment of toxicity:}

Assessment of toxicity was done by FAF-Drugs web-server Comparison

\section{of the ligand with other inhibitors:}

This structure was compared with 11 other compounds to see the interaction with 47 important enzymes of the human body using Autodock Vina.

Results: 
The enzyme reverse transcriptase was searched in protein data bank website and the results revealed 382 proteins of different species among them 53 proteins were associated with Homo sapiens. The first structure to arrive was the crystal structure of human Importin alpha 5 with TERT (Telomerase Reverse Transcriptase) NLS (Nuclear Localisation Sequence) peptide with PDB ID 4B18 released on $4^{\text {th }}$ of September 2013. The structure was predicted using X-Ray diffraction with resolution of $2.52 \AA$. The compound had two polymers. The first molecule was importin subunit alpha- 1 which is a protein with 447 amino acids isolated from Homo sapiens. The second molecule was telomerase reverse transcriptase which was also a protein with 20 amino acids isolated from

Homo sapiens. After downloading this protein, it was observed that although in the website it indicated that the fragment consists of residues from 222-240 but when visualizing the protein, it showed that 4 amino acids from 225-228 were absent. So to complete the protein structure, the crystal structure of human Importin alpha 5 with TERT NLS peptide was searched in protein database of PubMed, the FASTA format of this protein was found and missing amino acids were inserted using discovery studio.

The 3D structure of telomerase reverse transcriptase is shown below:

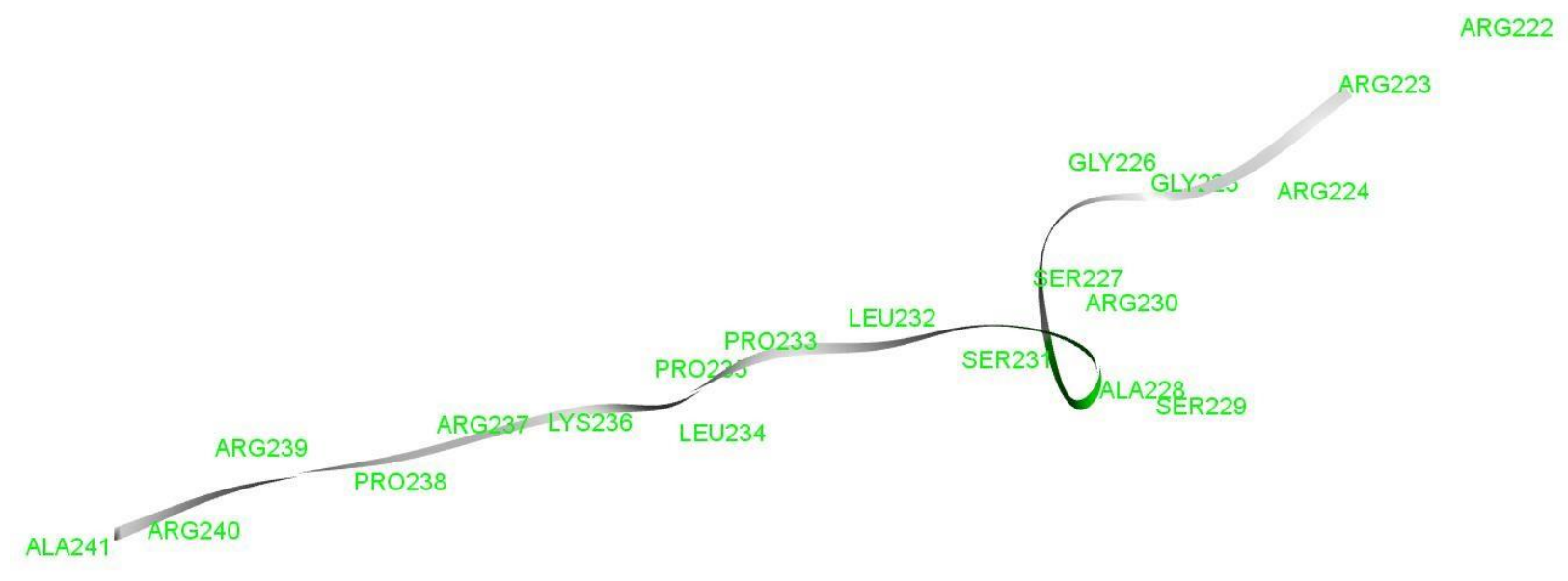

Figure 1: 3D structure of telomerase reverse transcriptase showing amino acid residues

The amino acid sequence of telomerase reverse transcriptase is RRRGGSASRSLPLPKRPRRA. Alpha tocopherol was modified into 289 structures and compound 214 showed the highest binding affinity with a binding energy of $-7.5 \mathrm{Kcal} / \mathrm{mol}$. 


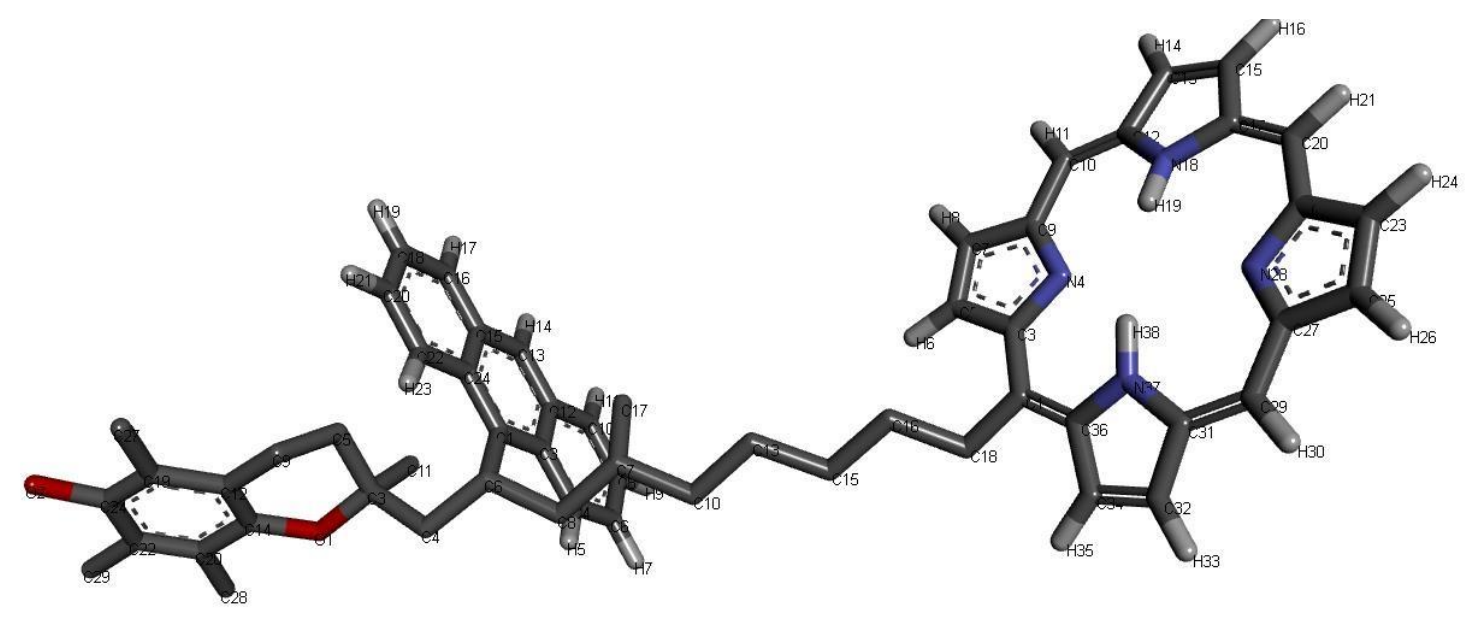

Figure 2: Structure of compound 214 showing carbon atoms.

Ligand

van der Waals force

Hydrogen Bond

Pi-cation interactions

Figure 3: Compound 214 binding with telomerase reverse transcriptase by Vanderwaals forces and

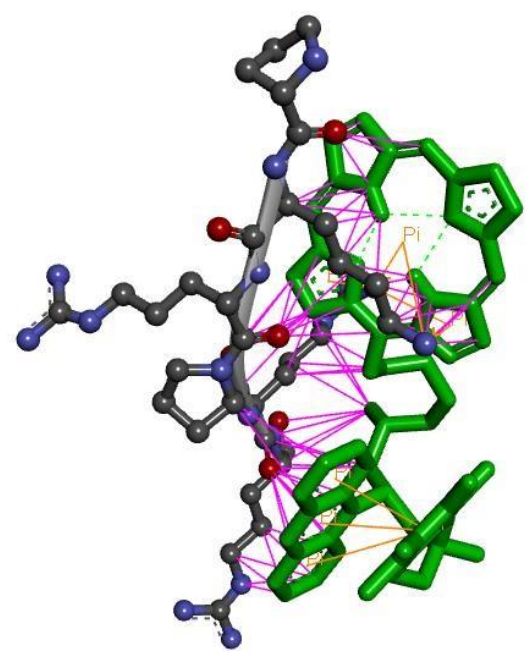
pication interactions

\section{Assessment of toxicity:}

Toxicity was assessed using FAF-Drugs web server which revealed that the drug is non-toxic. 


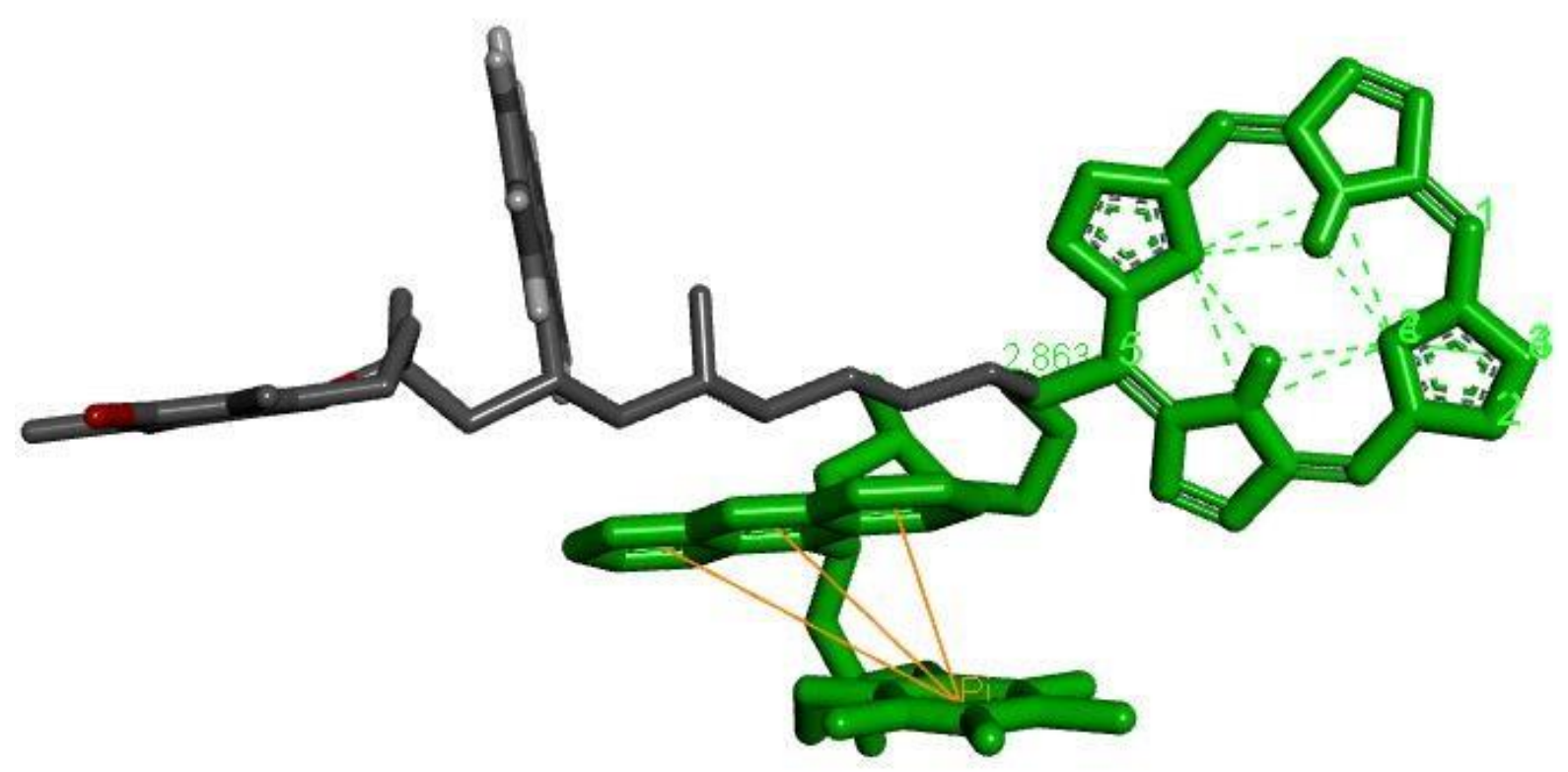

Figure 4: The bounded compound 214 when superimposed with the starting molecule on porphyrin rings also shows pi-pi interactions (yellow lines) between the aromatic ring and the anthryl group in $6^{\text {th }}$ position and formation of hydrogen bonds (green dotted line) within the porphyrin ring

\section{Comparison of compound $\mathbf{2 1 4}$ with other telomerase reverse transcriptase inhibitors currently under research:}

The following table shows that compound 214 shows the lowest binding energy with telomerase reverse transcriptase compared to other inhibitors currently under research.

Table 1: Binding energy of compound 214 and other telomerase reverse transcriptase inhibitors currently under research with telomerase reverse transcriptase

\begin{tabular}{cc}
\hline Structure & Binding Energy (Kcal/mol) \\
\hline Epigallocathechin & -4.7 \\
Tretinoin & -4.7 \\
Alterperilenol & -5.0 \\
Compound 214 & -7.5 \\
Curcumin & -4.6 \\
Dideoxyguanosinetriphosphate & -3.7 \\
Genistein & -4.5 \\
Helenalin & -4.9 \\
Isoprenoid & -4.0 \\
Levofloxacin & -4.8 \\
Ofloxacin & -4.7
\end{tabular}


Figure 88: All ligands are binding at a common site in telomerase reverse transcriptase.

Figure 5: Compound 214 and other inhibitors of telomerase reverse transcriptase binding to telomerase reverse transcriptase at the same site.

Comparison of compound 214 with other telomerase reverse transcriptase inhibitors currently under research with other important enzymes in the body:

The following tables show the interaction of other important enzymes of the body with compound 214 and other inhibitors currently under research. 
Table 2: Binding energy (in $\mathrm{kcal} / \mathrm{mol}$ ) of other inhibitors currently under research compared with compound 214 on oxidoreductases:

\begin{tabular}{|c|c|c|c|c|c|c|c|c|}
\hline \multirow[t]{2}{*}{ Enzyme inhibitors } & \multicolumn{8}{|c|}{ Oxidoreductase enzymes present in the human body (PDB ID) } \\
\hline & $\begin{array}{c}\text { Superoxide } \\
\text { dismutase } \\
(1 \mathrm{HL} 5)\end{array}$ & $\begin{array}{l}\text { Catalase } \\
\text { (1F4J) }\end{array}$ & $\begin{array}{c}\text { Glutathione S } \\
\text { Transferase } \\
(2 \mathrm{VCV})\end{array}$ & $\begin{array}{c}\text { Glyceraldehyde } 3 \\
\text { Phosphate } \\
\text { Dehydrogenase } \\
\text { (1ZNQ) }\end{array}$ & $\begin{array}{c}\text { Succinate } \\
\text { dehydrogenase } \\
(2 \mathrm{~W} 8 \mathrm{R})\end{array}$ & $\begin{array}{c}\text { Cyt } \\
\text { P450 } \\
1 \mathrm{~A} 1 \\
(418 \mathrm{~V})\end{array}$ & $\begin{array}{c}\text { Pyruvate } \\
\text { dehydrogenase } \\
\text { (3EXE) }\end{array}$ & $\begin{array}{c}\text { Aldehyde } \\
\text { dehydrogenase } \\
\text { (2ONM) }\end{array}$ \\
\hline Epigallocatechin & -7.9 & -9.2 & -7.8 & -9.5 & -8.1 & -7.5 & -8.2 & No affinity \\
\hline Curcumin & -7.6 & -8.1 & -7.7 & -8.0 & -7.6 & -9.5 & -7.9 & No affinity \\
\hline Genistein & -7.2 & -9.6 & -7.8 & -8.3 & -7.8 & -10.4 & -7.9 & No affinity \\
\hline Isoprenoid & -5.1 & -6.3 & -5.9 & -6.0 & -5.9 & -5.2 & -5.9 & -5.0 \\
\hline Tretinoin & -6.9 & -7.8 & -7.8 & -7.0 & -7.5 & -6.9 & -7.2 & No affinity \\
\hline Zidovudine & -7.5 & -8.6 & No affinity & No affinity & -7.2 & -7.3 & No affinity & No affinity \\
\hline Ofloxacin & -7.5 & -9.2 & -8.0 & -7.7 & -8.3 & -7.3 & -8.0 & No affinity \\
\hline Levofloxacin & -7.9 & -8.2 & -7.9 & -8.5 & -8.3 & -8.3 & -7.3 & No affinity \\
\hline Alterperilenol & -8.2 & -10.8 & -8.6 & -9.0 & -9.0 & -8.0 & -10.1 & No affinity \\
\hline $\begin{array}{c}\text { Dideoxyguanosinetr } \\
\text { iphosphate }\end{array}$ & -7.0 & -8.6 & No affinity & No affinity & -7.5 & -7.5 & No affinity & No affinity \\
\hline Helenin & -7.9 & -8.0 & -7.7 & $-9.1-8.5$ & -6.8 & -7.4 & -7.2 & No affinity \\
\hline Compound 214 & -10.2 & -11.1 & -8.9 & -11.1 & -10.9 & -8.7 & -10.8 & No affinity \\
\hline
\end{tabular}


Compound 214 has been shown to bind with superoxide dismutase, catalase, glutathione s transferase, glyceraldehyde 3 phosphate dehydrogenase, succinate dehydrogenase, pyruvate dehydrogenase with highest binding affinity compared to other inhibitors currently under research. Though it interacted with Cyt P450 1A1 but its affinity was less than curcumin and genistein. Like other inhibitors it had no affinity with aldehyde dehydrogenase. 


\begin{tabular}{|c|c|c|c|c|c|c|c|c|c|}
\hline \multirow[b]{2}{*}{ Enzyme inhibitors } & \multicolumn{9}{|c|}{ Transferase enzymes present in the human body (PDB ID) } \\
\hline & $\begin{array}{l}\text { Pyruvat } \\
\text { e } \\
\text { Kinase } \\
\text { (1TSF) }\end{array}$ & $\begin{array}{c}\text { DNA } \\
\text { Polymera } \\
\text { se (3EOJ) }\end{array}$ & $\begin{array}{l}\text { Glucokina } \\
\text { se (1V4T) }\end{array}$ & $\begin{array}{l}\text { Phosphoglycer } \\
\text { ate kinase } \\
\text { (3C39) }\end{array}$ & $\begin{array}{c}\text { Acetyl } \\
\text { CO-A } \\
\text { Acetyl } \\
\text { transfera } \\
\text { se (2F2S) }\end{array}$ & $\begin{array}{c}\text { Serine } \\
\text { Threonine } \\
\text { Protein KInase } \\
(316 W)\end{array}$ & $\begin{array}{c}\text { Phosphatid } \\
\text { yl inositol } 3 \\
\text { kinase } \\
\text { (3CSF) }\end{array}$ & $\begin{array}{c}\text { UDP-N- } \\
\text { Acetylglucosa } \\
\text { mine (3PE3) }\end{array}$ & $\begin{array}{c}\text { Epiderma } \\
\text { I Growth } \\
\text { Factor } \\
\text { (3IEI) }\end{array}$ \\
\hline Epigallo Catechin & -8.6 & -7.3 & -7.3 & -6.9 & -7.5 & No affinity & -8.2 & -8.0 & -8.0 \\
\hline Curcumin & -7.9 & -7.1 & -6.5 & -6.7 & -6.1 & No affinity & -7.3 & -7.2 & -8.3 \\
\hline Genistein & -8.0 & -7.8 & -7.2 & -8.3 & -6.9 & No affinity & -9.0 & -7.2 & -8.4 \\
\hline Isoprenoid & -6.7 & -6.2 & -4.4 & -5.0 & -6.2 & -5.8 & -6.4 & -5.5 & -5.9 \\
\hline Tretinoin & -8.1 & -6.9 & -6.4 & -7.5 & -6.5 & No affinity & -7.6 & -7.9 & -8.4 \\
\hline Zidovudine & -8.0 & -6.9 & -6.4 & -6.2 & -6.9 & No affinity & No affinity & No affinity & -7.6 \\
\hline Ofloxacin & -8.4 & -8.0 & -7.2 & -7.1 & -7.3 & No affinity & -7.7 & No affinity & -7.5 \\
\hline Levofloxacin & -8.7 & -7.5 & -7.1 & -6.9 & -7.2 & No affinity & -8.1 & No affinity & -8.2 \\
\hline Alterperilenol & -8.9 & -7.9 & -7.6 & -8.7 & -7.9 & No affinity & -10.0 & -8.3 & -8.4 \\
\hline $\begin{array}{c}\text { Dideoxyguanosinetr } \\
\text { iphosphate }\end{array}$ & -9.5 & -6.5 & -7.0 & -8.6 & -6.3 & No affinity & No affinity & No affinity & -8.5 \\
\hline Helenin & -8.7 & -6.9 & -7.3 & -6.6 & -6.4 & No affinity & -7.8 & -7.5 & -6.8 \\
\hline
\end{tabular}


Compound 214 has been shown to bind with DNA polymerase, glucokinase, phosphoglycerate kinase, acetyl CO-A acetyl transferase, phosphatidyl inositol 3 kinase, UDP-N-Acetylglucosamine, epidermal growth factor with highest binding affinity compared to other inhibitors currently under research. Though it interacted with pyruvate kinase but its affinity was less than epigallocatechin, ofloxacin, levofloxacin, alterperilenol, dideoxyguanosinetriphosphate, and helenin. Like other inhibitors it had no affinity with Serine Threonine Protein Kinase. 
Table 4: Binding energy (in kcal/mol) of other inhibitors currently under research compared with compound 214 on hydrolases:

\begin{tabular}{|c|c|c|c|c|c|c|c|c|c|}
\hline \multirow{2}{*}{$\begin{array}{l}\text { Enzyme } \\
\text { Inhibitors }\end{array}$} & \multicolumn{9}{|c|}{ Hydrolase enzymes present in human body (PDB ID) } \\
\hline & $\begin{array}{c}\text { Tyrosine } \\
\text { Phosphatas } \\
\text { e (2JJD) }\end{array}$ & $\begin{array}{l}\text { Caspase } \\
\text { (3V6M) }\end{array}$ & $\begin{array}{l}\text { Dipeptidyl } \\
\text { Peptidase } \\
(3 C C B)\end{array}$ & $\begin{array}{l}\text { Ribonuclease } \\
\text { (3PUF) }\end{array}$ & $\begin{array}{c}\text { Phosphodiesterase } \\
\text { 4D (1MKD) }\end{array}$ & $\begin{array}{c}\text { Sucrase } \\
\text { isomaltase } \\
\text { (3LPO) }\end{array}$ & $\begin{array}{l}\text { Insulin } \\
\text { degrading } \\
\text { enzyme } \\
\text { (3OFI) }\end{array}$ & $\begin{array}{l}\text { Phosphatidyl } \\
\text { inositol } \\
\text { Phosphatase } \\
\text { (1W8O) }\end{array}$ & $\begin{array}{c}\text { Glutamate } \\
\text { Carboxypeptidase } \\
\text { (1Z8L) }\end{array}$ \\
\hline EpigalloCatechin & -8.1 & -8.1 & -9.3 & No affinity & -8.8 & -7.2 & -9.2 & -7.0 & -9.5 \\
\hline Curcumin & -8.0 & -7.2 & -7.8 & -6.9 & -8.6 & -7.5 & -8.8 & -6.7 & -9.3 \\
\hline Genistein & -7.5 & -7.6 & -9.2 & -7.8 & -9.3 & -6.7 & -8.6 & -6.8 & -8.3 \\
\hline Isoprenoid & -5.1 & -6.0 & -5.4 & -5.6 & -6.9 & -5.8 & -5.7 & -5.0 & -6.1 \\
\hline Tretinoin & -6.2 & -6.7 & -7.3 & No affinity & -8.4 & -7.5 & -8.6 & -6.6 & -8.1 \\
\hline Zidovudine & -7.0 & -7.3 & -7.7 & No affinity & No affinity & -6.4 & -7.5 & -7.4 & -8.2 \\
\hline Ofloxacin & -7.9 & -7.2 & -7.2 & No affinity & No affinity & -7.4 & -8.6 & -7.0 & -7.8 \\
\hline Levofloxacin & -7.5 & -6.9 & -8.1 & No affinity & No affinity & -7.5 & -8.0 & -7.0 & -8.2 \\
\hline Alterperylenol & -8.1 & -8.5 & -8.7 & No affinity & -10.1 & -8.1 & -9.8 & -7.5 & -9.6 \\
\hline $\begin{array}{l}\text { Dideoxyguanosin } \\
\text { etriphosphate }\end{array}$ & -7.2 & -6.9 & -8.1 & No affinity & No affinity & -7.9 & -8.6 & -7.0 & -9.9 \\
\hline Helenin & -7.1 & -6.7 & -7.3 & -7.4 & -8.1 & -7.2 & -7.5 & -7.4 & -8.8 \\
\hline Compound 214 & -10.3 & -9.9 & -11.6 & No affinity & -13.1 & No affinity & -10.8 & -8.2 & -12.8 \\
\hline
\end{tabular}


Compound 214 has been shown to bind with Tyrosine Phosphatase, Caspase, Dipeptidyl Peptidase,

Phosphodiesterase 4D, Insulin degrading enzyme, Phosphatidyl inositol Phosphatase, Glutamate Carboxypeptidase with highest binding affinity compared to other inhibitors currently under research. It had no affinity with ribonuclease and sucrase isomaltase 
Table 5: Binding energy (in kcal/mol) of other inhibitors currently under research compared with compound 214 on lyases:

\begin{tabular}{|c|c|c|c|c|c|c|c|c|}
\hline \multirow[t]{2}{*}{ Enzyme Inhibitor } & \multicolumn{8}{|c|}{ Lyase enzymes present in human body (PDB ID) } \\
\hline & $\begin{array}{l}\text { Enolase } \\
\text { (2PSN) }\end{array}$ & $\begin{array}{c}\text { Cyatathionine } \\
\text { beta synthase } \\
\text { (1JBQ) }\end{array}$ & $\begin{array}{l}\text { UDP glucoronic } \\
\text { acid } \\
\text { decarboxylase } \\
\text { (4LK3) }\end{array}$ & $\begin{array}{c}\text { DNA } \\
\text { lyase } \\
(3 \cup 8 U)\end{array}$ & $\begin{array}{c}\text { Hydroxymethyl } \\
\text { glutaryl-CoA lyase } \\
\text { (2CW6) }\end{array}$ & $\begin{array}{c}\text { Serine } \\
\text { dehydratase } \\
(2 \mathrm{RKB})\end{array}$ & $\begin{array}{c}\text { GDP } \\
\text { mannose } \\
\text { dehydratase } \\
(1 \mathrm{~T} 2 \mathrm{~A})\end{array}$ & $\begin{array}{c}\text { Ferrochelatase } \\
(2 \mathrm{HRE})\end{array}$ \\
\hline Epigallocatechin & -7.5 & -7.7 & -8.8 & -8.6 & -8.6 & -8.6 & -8.3 & -8.9 \\
\hline Curcumin & -7.7 & -6.5 & -7.4 & -6.8 & -8.4 & -7.7 & -9.6 & -8.2 \\
\hline Genistein & -7.3 & -7.4 & -8.3 & -8.0 & -8.3 & -7.8 & -9.8 & -8.5 \\
\hline Isoprenoid & -5.2 & -5.5 & -5.7 & -5.8 & -6.6 & -6.5 & -5.8 & -5.8 \\
\hline Tretinoin & -7.7 & -7.2 & -7.0 & -7.9 & -8.8 & -8.3 & -8.6 & -7.4 \\
\hline Zidovudine & -7.3 & No affinity & -7.4 & -6.8 & -7.4 & -8.2 & -9.0 & -7.8 \\
\hline Ofloxacin & -7.3 & -7.5 & -7.9 & -9.0 & -8.2 & -8.4 & -8.7 & -9.3 \\
\hline Levofloxacin & -7.6 & -6.5 & -8.7 & -8.6 & -8.9 & -8.7 & -9.5 & -9.0 \\
\hline Alterperylenol & -8.4 & -8.0 & -9.3 & -8.5 & -10.0 & -9.8 & -8.4 & -10.0 \\
\hline $\begin{array}{c}\text { Dideoxyguanosin } \\
\text { etriphosphate }\end{array}$ & -7.6 & No affinity & -9.1 & -8.1 & -8.5 & -9.1 & -10.0 & -7.6 \\
\hline Helenin & -7.2 & -7.6 & -8.5 & -7.6 & -9.9 & -8.3 & -8.9 & -7.8 \\
\hline Compound 214 & -10.0 & -9.8 & -10.8 & -10.4 & -13.3 & -12.3 & -9.2 & -10.2 \\
\hline
\end{tabular}


Compound 214 has been shown to bind with Enolase, Cyatathionine beta synthase, UDP glucoronic acid decarboxylase, DNA lyase, Hydroxymethyl glutaryl-CoA lyase, Serine dehydratase and

Ferrochelatase with highest binding affinity compared to other inhibitors currently under research. Though it interacted with GDP mannose dehydratase but its affinity was less than curcumin, levofloxacin, dideoxyguanosinetriphosphate, and genistein 
Table 6: Binding energy (in $\mathrm{kcal} / \mathrm{mol}$ ) of other inhibitors currently under research compared with compound 214 on isomerases:

\begin{tabular}{|c|c|c|c|c|c|c|}
\hline \multirow{2}{*}{ Enzyme inhibitors } & \multicolumn{6}{|c|}{ Isomerase enzymes present in human body (PDB ID) } \\
\hline & $\begin{array}{c}\text { Glucose } 6 \\
\text { Phosphate } \\
\text { Isomerase } \\
\text { (1JLH) }\end{array}$ & $\begin{array}{c}\text { Triose } \\
\text { phosphate } \\
\text { isomerase } \\
\text { (4BR1) }\end{array}$ & $\begin{array}{c}\text { Protein } \\
\text { Phosphatase } \\
\text { (2JJD) }\end{array}$ & $\begin{array}{c}\text { Autocrine } \\
\text { Motility } \\
\text { Factor (1IRI) }\end{array}$ & $\begin{array}{l}\text { Biphospho } \\
\text { glycerate } \\
\text { mutase } \\
\text { (3NFY) }\end{array}$ & $\begin{array}{l}\text { UDP N-Acetyl } \\
\text { glucosamine } 2 \\
\text { epimerase } \\
\text { (3EO3) }\end{array}$ \\
\hline EpigalloCatechin & -7.3 & -8.7 & -8.3 & -8.9 & -7.4 & -4.9 \\
\hline Curcumin & -6.8 & -8.0 & -7.7 & -7.2 & -7.4 & -6.7 \\
\hline Genistein & -7.6 & -8.5 & -8.1 & -7.8 & -8.2 & -6.6 \\
\hline Isoprenoid & -4.7 & -5.8 & -5.5 & -5.3 & -5.5 & -4.7 \\
\hline Tretinoin & -6.5 & -7.5 & -7.3 & -6.9 & -7.0 & -5.0 \\
\hline Zidovudine & No affinity & -7.8 & -8.3 & No affinity & -7.2 & -4.9 \\
\hline Ofloxacin & No affinity & -7.7 & -7.7 & -7.0 & -8.3 & -4.8 \\
\hline Levofloxacin & No affinity & -7.5 & -8.5 & -6.7 & -8.2 & -5.1 \\
\hline Alterperylenol & -8.4 & -7.7 & -9.1 & -8.4 & -8.2 & -5.5 \\
\hline $\begin{array}{c}\text { Dideoxyguanosinetri } \\
\text { phosphate }\end{array}$ & No affinity & -9.0 & -8.3 & No affinity & -8.8 & -4.2 \\
\hline Helenin & -7.5 & -6.6 & -7.6 & -7.9 & -7.0 & -5.4 \\
\hline Compound 214 & -9.9 & -7.3 & -10.0 & -10.2 & -8.3 & -6.4 \\
\hline
\end{tabular}


Compound 214 has been shown to bind with Glucose 6 Phosphate Isomerase, Protein Phosphatase, Autocrine Motility Factor and Biphospho glycerate mutase with highest binding affinity compared to other inhibitors currently under research. Though it interacted with Triose phosphate isomerase and UDP N-Acetyl glucosamine 2 epimerase but its affinity was not highest. 
Table 7: Binding energy (in $\mathrm{kcal} / \mathrm{mol}$ ) of other inhibitors currently under research compared with compound 214 on ligases:

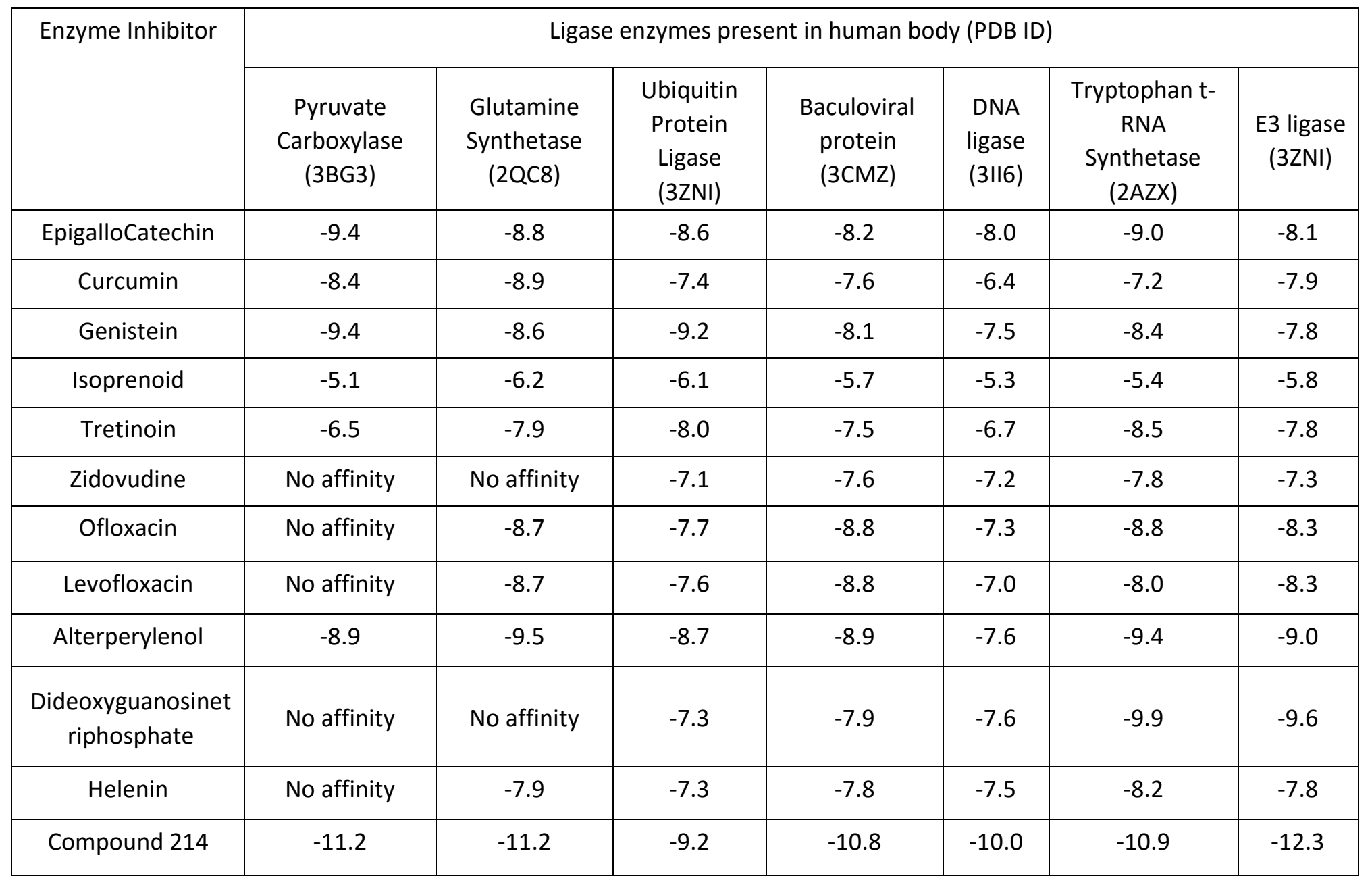


Compound 214 has been shown to bind with Pyruvate Carboxylase, Glutamine Synthetase, Ubiquitin Protein Ligase, Baculoviral protein, DNA ligase, Tryptophan t-RNA Synthetase and E3 ligase with highest binding affinity compared to other inhibitors currently under research.

\section{Discussion:}

The in silico voyage of discovering a cure for arsenicosis resulted in rational designing of a drug which binds with telomerase reverse transcriptase. Telomerases are part of a distinct subgroup of RNAdependent polymerases. Telomerase lengthens telomeres in DNA strands, thereby allowing senescent cells that would otherwise become postmitotic and undergo apoptosis and become potentially immortal cancerous cells. To be specific, telomerase reverse transcriptase is responsible for catalyzing the addition of nucleotides in a TTAGGG sequence to the ends of a chromosome's telomeres

[12]. This addition of repetitive DNA sequences prevents degradation of the chromosomal ends following multiple rounds of replication [20].

Genome-wide association studies suggest telomerase reverse transcriptase is a susceptibility gene for development of many cancers including lung cancer. Telomerase activity is associated with the number of times a cell can divide playing an important role in the immortality of cell lines, such as cancer cells. The enzyme complex acts through the addition of telomeric repeats to the ends of chromosomal DNA.

This generates immortal cancer cells [17]. In fact, there is a strong correlation between telomerase activity and malignant tumors or cancerous cell lines [17]. Not all types of human cancer have increased telomerase activity. $90 \%$ of cancers are characterized by increased telomerase activity [17]. Lung cancer is the most well characterized type of cancer associated with telomerase.

If increased telomerase activity is associated with malignancy, then possible cancer treatments could involve inhibiting its catalytic component, human telomerase reverse transcriptase, to reduce the enzyme's activity and cause cell death. Since normal somatic cells do not express telomerase reverse

transcriptase, telomerase inhibition in cancer cells can cause senescence and apoptosis without affecting normal human cells [17]. Inhibition of telomerase reverse transcriptase led to apoptosis and cell death in cells with short telomere lengths a promising result for cancer treatment [17]. Although cells with long telomeres did not experience apoptosis, they developed mortal characteristics and underwent telomere shortening [17].

\section{Conclusion:}

The findings reveal that if many drugs are proven effective for a disease whose exact molecular pathogenesisis not clearly defined, then those drugs have the possibility of binding to a common target

to alleviate the symptoms. This study also suggests the rationality of using these compounds in combination so long as an effective cure is not discovered for arsenicosis. The minerals zinc and selenium may be explored in the future if metal docking softwares are developed. Identification of active compounds in the food stuffs proven to be effective in this condition and their in silico analysis may disclose more insights in this field. The potential areas of in silico intervention in the drug discovery process expose endless possibilities and opportunities for research at a molecular level.

\section{Declaration:}


Availability of data and materials: The data supporting findings can be found within the manuscript.

\section{Author Contributions:}

None of the authors have any competing interests. Tarek Golam Mustafa Mohammod conceived of the presented idea, developed the theory. performed the computations and verified the analytical methods.

Mir Misbahuddin encouraged Tarek Golam Mustafa Mohammod to investigate and supervised the findings of this work. All authors discussed the results and contributed to the final manuscript.

\section{Acknowledgement:}

The authors acknowledge Bangabandhu Sheikh Mujib Medical University for conducting the research.

\section{Reference:}

1. Alland, C, Moreews, F, Boens, D, Carpentier, M, Chiusa, S, Lonquetly, M et al. 2005 m 'ROVSL a web resource for structural bioinformatics' Nucleic Acids Research Vol. 1: pp. 44-49.

2. Altschul, SF, Gish, W, Miller, W, Myers, EW \& Lipman, DJ, 1990, 'Basic local alignment search tool' Journal of Molecular Biology Vol. 215: pp. 403-410.

3. Umar, BU, 2007, 'Effect of hexane extract of spinach in the removal of arsenic from rat6' Bangladesh Journal of Pharmacology Vol. 2: pp. 24-34

4. Noor Jahan Alam Chowdhury, Mir Misbahuddin and Md. Sayedur Rahman Corn extracts lower tissue arsenic level in rat Bangladesh Med Res Counc Bull 2009; 35: 21-25

5. Erika García-Chávez, Ismael Jiménez, Bertha Segura, Luz M. Del Razo, Lipid oxidative damage and distribution of inorganic arsenic and its metabolites in the rat nervous system after arsenite exposure:

Influence of alpha tocopherol supplementation, NeuroToxicology, Volume 27, Issue 6, 2006, Pages 10241031

6. Hall, AF,1946, 'Arsenical keratosis disappearing with vitamin A therapy'Archives of Dermatology and Syphilology Vol. 53: pp. 154

7. Kaufmann, RB, Sorensen, BH, Rahman, M, Streatfield, K \&Persson, LA, 2001,'Addressing the public health crisis caused by arsenic contamination of drinking water in Bangladesh' Dhaka, World Bank, pp 1-30.

8. Kamaluddin M, Misbahuddin M. Zinc supplement on tissue arsenic concentration in rats. Bangladesh Med Res Counc Bull. 2006 Dec;32(3) 87-91.

9. Misbahuddin M, Maidul Islam AZM, Khandker S, Ifthaker AI M, Islam N, Anjumanara. Efficacy of Spirulina Extract Plus Zinc in Patients of Chronic Arsenic Poisoning: A Randomized PlaceboControlled Study. Clinical Toxicology. 2006;44(2):135-41.

10. Mir Misbahuddin, Tahmina Bashar and Md. Amir Hossain Effectiveness of garlic oil in the treatment of arsenical palmar keratosis Bangladesh J Pharmacol 2013; 8: 22-27 
11. Md. Faizur Rahman and Mir Misbahuddin Bangladesh Effect of folic acid and tetrahydrofolate on tissue arsenic level in rat J Pharmacol 2010; 5: 25-29

12. Poole, JC, Andrews, LG \& Tollefsbol, TO, 2001, 'Activity, functin and gene regulation of the catalytic submit of telomerase (hTERT)' Gene Volo. 269: pp. 1-12

13. SAHA, Beauty. Effect of ascorbic acid on reduced glutathione level in arsenic-loaded isolated liver tissues of rat. Bangladesh Journal of Pharmacology, [S.I.], v. 1, n. 2, p. 68-71, Dec. 2006.

14. Schneider, G \& Fechner, U, 2005, 'Computer-based de novo design of drug-like molecules' Nature Reviews Drug Discovery Vol. 4: pp. 649-66.

15. Shampay, J \& Blackbur, EH, 1988, 'Generation of Telomere-length heterogeneity in Saccharomyces cerevisiae' Proceedings of the national Academy of Science Vol. 85: pp. 534-8.

16. Miroslav Styblo, David J. Thomas, Selenium Modifies the Metabolism and Toxicity of Arsenic in Primary Rat Hepatocytes, Toxicology and Applied Pharmacology, Volume 172, Issue 1, 2001, Pages 52-61

17. Sundin, T \& Hentosh, P 2012, 'InTERTesting association between telomerase, mTOR and phytochemicals' Expert Reviews Molecular Medicine Vol. 14: pp. e8.

18. Tabassum, N. E. "Effect of alpha-lipoic acid on the removal of arsenic from arsenic-loaded isolated liver tissues of rat." Bangladesh J Pharmacol 1 (2006): 27-32.

19. Wang, R, Gao, Y \& Lai, L, 2000, 'LigBuilder: A Multi-Purpose Program for Structure-Based Drug Design' Journal of Molecular Modeling Vol. 6: pp. 498-516.

20. Zhang, X, Mar, V, Zhou, W, Harrington, L \& Robinson, MO, 1999, 'Telomere shortening and apoptosis in telomerase-inhibited human tumor cells' Genes Development Vol. 13: pp. 2388-99 